\title{
APPROXIMATING THE KOHLRAUSCH FUNCTION BY SUMS OF EXPONENTIALS
}

\author{
MIN ZHONG ${ }^{\otimes 1}$, R. J. LOY ${ }^{2}$ and R. S. ANDERSSEN ${ }^{3}$
}

(Received 26 April, 2012; revised 6 November, 2012)

\begin{abstract}
The Kohlrausch functions $\exp \left(-t^{\beta}\right)$, with $\beta \in(0,1)$, which are important in a wide range of physical, chemical and biological applications, correspond to specific realizations of completely monotone functions. In this paper, using nonuniform grids and midpoint estimates, constructive procedures are formulated and analysed for the Kohlrausch functions. Sharper estimates are discussed to improve the approximation results. Numerical results and representative approximations are presented to illustrate the effectiveness of the proposed method.
\end{abstract}

2010 Mathematics subject classification: primary 65D20; secondary 28E99, 33F05, $65 \mathrm{R} 10$.

Keywords and phrases: Kohlrausch function, sums of exponentials, approximation.

\section{Introduction}

The set of completely monotone (CM) functions includes many interesting examples which arise in the modelling of real-world problems [5, 10, 13, 14]. An important practical example of such functions are the Kohlrausch (Williams-Watts, stretched exponential) functions,

$$
\exp \left(-\alpha t^{\beta}\right), \quad \alpha>0,0<\beta<1, t \geq 0 .
$$

Such functions have important mathematical properties [5], and when used as empirical decay laws, arise in a broad spectrum of applications, including the modelling of the glassy state of dense matter, nonexponential correlation functions in nuclear magnetic resonance, polymer dynamics, and bone and muscle rheology $[8,15$, 16, 20-22]. Because of their practical importance, there is a need to have appropriate approximations for them.

\footnotetext{
${ }^{1}$ School of Mathematical Sciences, Fudan University, 200433 Shanghai, People's Republic of China; e-mail: 09110180007@fudan.edu.cn.

${ }^{2}$ Mathematical Sciences Institute, Australian National University, Canberra, ACT 0200, Australia; e-mail: rick.loy@anu.edu.au.

${ }^{3}$ CSIRO Mathematics, Informatics and Statistics, GPO Box 664, Canberra, ACT 2601, Australia; e-mail: bob.anderssen@csiro.au.

(C) Australian Mathematical Society 2013, Serial-fee code 1446-1811/2013\$16.00
} 
For example [1-4], in rheological applications, though the relaxation and creep modulii $G(t)$ and $J(t)$ of linear viscoelasticity are known to satisfy the interconversion equation

$$
\int_{0}^{t} G(t-\tau) J(\tau) d \tau=\int_{0}^{t} J(t-\tau) G(\tau) d \tau=t, \quad t>0,
$$

$J(t)$ is unknown when $G(t)$ is a Kohlrausch function. A theoretical analysis shows that, for $G(t)$ a CM function, $J(t)$ must be a strictly monotonically increasing function with $\dot{J}(t)$ a CM function. It is well known that, for a sum of exponentials representation for $G(t)$, the corresponding analytical solution for $J(t)$ is again a sum of exponentials with a van der Monde matrix relationship defining the connection [17]. In addition, since Laplace transforms for the Kohlrausch functions are not known analytically, it would be useful to have approximations to them which could be utilized in applications. This leads naturally to the idea of approximating Kohlrausch functions by sums of CM functions for which the Laplace transforms are known. The obvious functions to choose are exponential functions.

Liu [11] proved in 2001 that if a function can be approximated arbitrarily closely by Dirichlet series with nonnegative coefficients in the supremum norm or $L^{p}$ norm with $p \in[1, \infty)$, then it must be a CM function. Conversely, any CM function can be so approximated. This raises the question about how such sums of exponentials could be constructed.

Pollard [19] proved in 1946 that, for a fixed $\beta$, the Kohlrausch function $\exp \left(-t^{\beta}\right)$ has the Laplace transform representation

$$
\exp \left(-t^{\beta}\right)=\int_{0}^{\infty} \phi(\beta, p) \exp (-p t) d p, \quad 0<\beta<1, t \geq 0,
$$

where

$$
\phi(\beta, p)=\frac{1}{\pi} \int_{0}^{\infty} \exp (-p u) \exp \left(-u^{\beta} \cos (\beta \pi)\right) \sin \left(u^{\beta} \sin (\beta \pi)\right) d u .
$$

This is the (stable) Lévy distribution $[9,15]$. An alternative integral representation for $\phi(\beta, p)$ is

$$
\phi(\beta, p)=\frac{1}{\pi} \int_{0}^{\infty} \exp \left(-u^{\beta} \cos (\beta \pi / 2)\right) \cos \left(u^{\beta} \sin (\beta \pi / 2)-p u\right) d u .
$$

This is given by Berberan-Santos et al. [6, 7], and also by Anderssen et al. [5], where there is an unfortunate sign error in the integrand. Independently, Penson and Górska [18] have investigated hypergeometric series representations for $\phi(\beta, p)$ when $\beta=l / k$ with $k$ and $l$ integers, $k>l$. However, truncations of these representations will not be CM, whereas the approximations proposed below and by Pollard [19] are automatically CM.

It is only for $\beta=1 / 2$ that a simple analytical expression is known in terms of elementary functions:

$$
\phi(1 / 2, p)=\int_{0}^{\infty} \exp (-p u) \sin \left(u^{1 / 2}\right) d u=\frac{\sqrt{\pi} \exp (-1 / 4 p)}{2 p^{3 / 2}} .
$$


Motivated by the Pollard [19] representation (1.2), Loy and Anderssen [12] constructed sums of exponentials approximations to those $\mathrm{CM}$ functions which are the Laplace transforms of positive absolutely continuous measures, and proved uniform convergence, and mean convergence under extra hypotheses. However, their results, based on the use of the integral mean value theorem, are purely theoretical. In this paper, by utilizing a nonuniform grid structure and sharper estimates for the required mean values, constructive procedures are formulated and analysed for the Kohlrausch functions.

The paper is organized as follows. The required background about CM functions, especially the Kohlrausch functions, is given in Section 2. A constructive procedure for the approximation of the Kohlrausch functions by sums of exponentials is given in Section 3. Error estimates are derived in the same section. Some numerical examples for different values of $\beta$ are given in Section 4. Nonuniform grids are considered for reducing the number of exponentials, and midpoint estimates for the mean value points are utilized. Sharper estimates for the mean value points are discussed in Section 5, where improved approximations are obtained. Some representative sums of exponentials approximations for different values of $\beta$ are given in Table 2 .

\section{Basic properties of the Kohlrausch functions}

The formal definition of CM functions is as follows.

Definition 2.1. A function $f:[0, \infty) \rightarrow[0, \infty)$ is completely monotone if it is $C^{\infty}$ on $(0, \infty)$, continuous at 0 and satisfies

$$
(-1)^{n} f^{(n)}(t) \geq 0, \quad t>0, n=0,1, \ldots
$$

The importance of CM functions is encapsulated in the following theorem.

Theorem 2.2 (Bernstein). A function $f:[0, \infty) \rightarrow[0, \infty)$ is CM if and only if there is a (unique) positive finite Borel measure $\mu$ on $[0, \infty)$ such that

$$
f(s)=\int_{0}^{\infty} \exp (-s t) d \mu(t), \quad s \geq 0 .
$$

The simplest $\mathrm{CM}$ functions are the exponentials $\exp (-\alpha t)$ for $\alpha \geq 0$, with Bernstein measures $\mu=\delta(t-\alpha)$, where $\delta(t-\alpha)$ denotes the Dirac point mass located at $t=\alpha$. The functions $\phi(\beta, p)$ arising from the Kohlrausch functions are nonnegative and have the following properties [4].

- For each $\beta \in(0,1)$,

$$
\int_{0}^{\infty} \phi(\beta, p) d p=1 .
$$

- For each $\beta \in(0,1)$, there exists a unique $p_{\beta}$ such that

$$
\phi\left(\beta, p_{\beta}\right)=\max _{p} \phi(\beta, p)=K_{\beta} .
$$


TABLE 1. Proportional contributions, in successive intervals of the independent variable $p$, to the area under $\phi(\beta, p)$.

\begin{tabular}{ccccccc}
\hline \multicolumn{7}{c}{$p$} \\
\hline$\beta$ & $\left(0,10^{-4}\right)(\%)$ & $\left(10^{-4}, 0.1\right)(\%)$ & $(0.1,1)(\%)$ & $(1,10)(\%)$ & $\left(10,10^{4}\right)(\%)$ & Total \\
\hline 0.9 & $<0.01$ & $<0.01$ & 63.2 & 35.3 & 1.4 & 99.9 \\
0.7 & $<0.01$ & $<0.01$ & 53.7 & 39.0 & 7.2 & 99.9 \\
0.5 & $<0.01$ & 2.5 & 45.4 & 34.4 & 17.1 & 99.4 \\
0.3 & $<0.01$ & 16.6 & 26.6 & 23.5 & 28.4 & 95.1 \\
0.1 & 8.8 & 21.3 & 8.5 & 8.4 & 21.5 & 68.5 \\
\hline
\end{tabular}

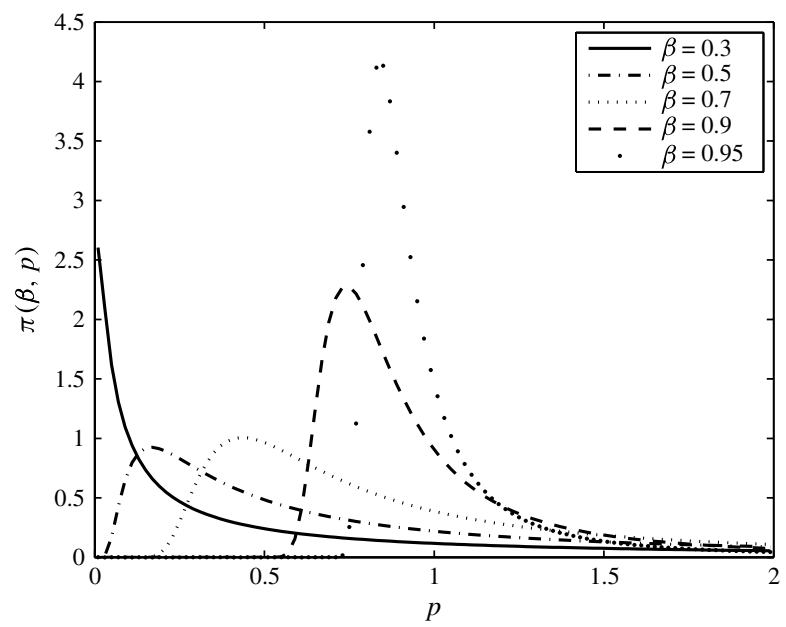

FIGURE 1. Representative plots of $\phi(\beta, p)$ for $\beta=0.3,0.5,0.7,0.8,0.9,0.95$.

- When $\beta$ is small, $\phi(\beta, p)$ has a long tail which is responsible for the associated fast initial decay in $\exp \left(-t^{\beta}\right)$. This is illustrated in Table 1 .

Some representative plots for $\phi(\beta, p)$ for different values of $\beta$ are given in Figure 1, which illustrates graphically the results of Table 1.

\section{Algorithms and error analysis}

For a positive integer $N>1$, the identity (1.1) can be rewritten in the following compartmentalized form:

$$
\exp \left(-t^{\beta}\right)=\Upsilon_{0}(t)+\sum_{i=1}^{N} \int_{p_{i}}^{p_{i+1}} \exp (-p t) \phi(\beta, p) d p+\Upsilon_{\infty}(t)
$$

where

$$
\Upsilon_{0}(t)=\int_{0}^{p^{\#}} \phi(\beta, p) \exp (-p t) d p, \quad \Upsilon_{\infty}(t)=\int_{p^{\# \#}}^{\infty} \phi(\beta, p) \exp (-p t) d p
$$


and

$$
0<p^{\#}(\beta)=p_{1}<p_{2}<\cdots<p_{N}<p_{N+1}=p^{\# \#}(\beta)<\infty
$$

is a given grid.

Lemma 3.1 (Loy and Anderssen [12]). For $t>0$ and any given $\epsilon>0$, there exist $p^{\# \#}>$ $p^{\#}>0$ such that

$$
\Upsilon_{0}(t)=\int_{0}^{p^{\#}} \phi(\beta, p) e^{-p t} d p<\epsilon, \quad \Upsilon_{\infty}(t)=\int_{p^{\# \#}}^{\infty} \phi(\beta, p) e^{-p t} d p<\epsilon .
$$

Since $\phi(\beta, p)$ is a nonnegative function, the integral mean value theorem can be applied to each term in the sum in (3.1). For each interval $\left[p_{i}, p_{i+1}\right]$, there exists the corresponding mean value point $p(i, t) \in\left(p_{i}, p_{i+1}\right)$ such that

$$
\exp (-p(i, t) t) \int_{p_{i}}^{p_{i+1}} \phi(\beta, p) d p=\int_{p_{i}}^{p_{i+1}} \exp (-p t) \phi(\beta, p) d p
$$

and hence

$$
\exp \left(-t^{\beta}\right)=\Upsilon_{0}(t)+\Upsilon_{\infty}(t)+\sum_{i=1}^{N} a_{i}^{*} \exp (-p(i, t) t)
$$

where

$$
a_{i}^{*}=\int_{p_{i}}^{p_{i+1}} \phi(\beta, p) d p
$$

In the analysis of Loy and Anderssen [12], any choice of $p_{i}^{*} \in\left(p_{i}, p_{i+1}\right)$, independent of $t$, as an approximation to $p(i, t)$, yielded uniform convergence for the approximation

$$
\sigma(\beta, N)(t)=\sum_{i=1}^{N} a_{i}^{*} \exp \left(-p_{i}^{*} t\right) \approx \sum_{i=1}^{N} a_{i}^{*} \exp (-p(i, t) t) .
$$

For the approximation $\sigma(\beta, N)(t)$ in (3.4), as well as the need to choose values for the $p_{i}^{*}$, an appropriate procedure is required for the evaluation of the coefficients $a_{i}^{*}$. For this it is necessary to complementarily utilize the two expressions for $\phi(\beta, p)$ in (1.2) and (1.3). The integral (1.2) is difficult to compute numerically for small values of $p$, owing to the rapid oscillations of the integrand, while the integral (1.3) is difficult to compute for large values of $p$, also owing to the rapid oscillations of the integrand.

Lemma 3.2. For a fixed $\beta \in(0,1)$, the integrand in the expression for $\phi(\beta, p)$ in (1.2),

$$
f_{1}(p, u)=\exp (-p u) \exp \left(-u^{\beta} \cos (\beta \pi)\right) \sin \left(u^{\beta} \sin (\beta \pi)\right)
$$

is integrable on the domain $\left[p_{i}, p_{i+1}\right] \times(0, \infty)$ with $0<p_{i}<p_{i+1}<\infty$. Consequently, the order of integration in (3.3) can be changed, that is,

$$
a_{i}^{*}=\int_{p_{i}}^{p_{i+1}} \int_{0}^{\infty} f_{1}(p, u) d u d p=\int_{0}^{\infty} \int_{p_{i}}^{p_{i+1}} f_{1}(p, u) d p d u .
$$


Proof. By Fubini's theorem, it is sufficient to prove that the integral

$$
\int_{0}^{\infty}\left(\int_{p_{i}}^{p_{i+1}}\left|f_{1}(p, u)\right| d p\right) d u
$$

is finite, and this can be divided into separate steps depending on the values of $\beta$. For fixed $\beta \in(0,0.5]$, we have $\cos (\beta \pi) \geq 0$ and hence

$$
\begin{aligned}
\int_{0}^{\infty} \int_{p_{i}}^{p_{i+1}}\left|f_{1}(p, u)\right| d p d u & \leq \int_{0}^{\infty} \int_{p_{i}}^{p_{i+1}} \exp (-p u) d p d u \\
& \leq\left(p_{i+1}-p_{i}\right) \int_{0}^{\infty} \exp \left(-p_{i} u\right) d u=\frac{p_{i+1}-p_{i}}{p_{i}} .
\end{aligned}
$$

For fixed $\beta \in(0.5,1)$, we have $\cos (\beta \pi)<0$. For $p \in\left[p_{i}, p_{i+1}\right]$, choose $q \in\left(0, p_{i}\right)$ and then $M$ sufficiently large so that, for all $u \geq M$,

$$
-u^{\beta} \cos (\beta \pi)-p_{i} u<-q u \text {. }
$$

Then

$$
\begin{aligned}
\int_{0}^{\infty} & \int_{p_{i}}^{p_{i+1}}\left|f_{1}(p, u)\right| d p d u \\
& \leq \int_{0}^{\infty} \int_{p_{i}}^{p_{i+1}} \exp \left(-u^{\beta} \cos (\beta \pi)-p u\right) d p d u \\
& \leq \int_{0}^{M} \int_{p_{i}}^{p_{i+1}} \exp \left(-u^{\beta} \cos (\beta \pi)-p u\right) d p d u+\int_{M}^{\infty} \int_{p_{i}}^{p_{i+1}} \exp (-q u) d p d u \\
& \leq\left(p_{i+1}-p_{i}\right)\left(\int_{0}^{M} \exp \left(-u^{\beta} \cos (\beta \pi)\right) d u+\int_{M}^{\infty} \exp (-q u) d u\right) \\
& \leq\left(p_{i+1}-p_{i}\right)\left(M \exp \left(-M^{\beta} \cos (\beta \pi)\right)+\frac{1}{q} \exp (-q M)\right)
\end{aligned}
$$

Combining these two bounds yields the result.

Using the expression for $\phi(\beta, p)$ in (1.2), the change in the order of integration in the formula for $a_{i}^{*}$ yields

$$
a_{i}^{*}=\Psi\left(p_{i}\right)-\Psi\left(p_{i+1}\right),
$$

where

$$
\Psi(p)=\frac{1}{\pi} \int_{0}^{\infty} \frac{\exp \left(-u^{\beta} \cos (\beta \pi)-p u\right) \sin \left(u^{\beta} \sin (\beta \pi)\right)}{u} d u
$$

Consequently, the evaluation of the $a_{i}^{*}$ is reduced to the evaluation of $\Psi(p)$.

In computation, in order to avoid the singular points of the integrand of $\Psi$, the following approximate integral is used:

$$
\Psi_{\epsilon, M}(p)=\frac{1}{\pi} \int_{\epsilon}^{M} \frac{\exp \left(-u^{\beta} \cos (\beta \pi)-p u\right) \sin \left(u^{\beta} \sin (\beta \pi)\right)}{u} d u .
$$


Theorem 3.3. Take $\beta \in(0,1)$ fixed and $\epsilon>0$. For a given $\delta>0$, there exists $M>0$ such that for all $p \geq \delta$, the uniform truncation error between $\Psi_{\epsilon, M}(p)$ and $\Psi(p)$ satisfies

$$
\left|\Psi_{\epsilon, M}(p)-\Psi(p)\right| \leq \exp \left(C_{\epsilon}\right) \epsilon^{\beta}+\frac{2 \exp (-M \delta / 2)}{M \delta \pi},
$$

where $C_{\epsilon}=\max \left\{0,-\epsilon^{\beta} \cos (\beta \pi)\right\}$ is the maximum value of $f(u)=-u^{\beta} \cos (\beta \pi)$ on $(0, \epsilon)$. Proof. The truncation error can be divided into two parts:

$$
\begin{aligned}
\left|\Psi_{\epsilon, M}(p)-\Psi(p)\right| & \leq \frac{1}{\pi}\left(\int_{0}^{\epsilon}+\int_{M}^{\infty}\right)\left|\frac{\exp \left(-u^{\beta} \cos (\beta \pi)-p u\right) \sin \left(u^{\beta} \sin (\beta \pi)\right)}{u}\right| d u, \\
& :=I_{1}+I_{2},
\end{aligned}
$$

where $I_{1}$ and $I_{2}$ denote the first and second integrals above, respectively. Recalling that $\sin \left(u^{\beta} \sin (\beta \pi)\right)<u^{\beta} \sin (\beta \pi)$ and $p>0$, the first integral can be estimated as

$$
I_{1} \leq \frac{\exp \left(C_{\epsilon}\right)}{\pi} \int_{0}^{\epsilon}\left|\frac{\sin (\beta \pi)}{u^{1-\beta}}\right| d u=\frac{\exp \left(C_{\epsilon}\right) \sin (\beta \pi) e^{\beta}}{\beta \pi} .
$$

The estimation of the bound for the second integral depends on the value of $\beta$. For $\beta \in(0.5,1)$, choose $M$ sufficiently large so that (3.5) is satisfied for $p \geq \delta$ with $q=\delta / 2$, that is, the inequality

$$
-u^{\beta} \cos (\beta \pi)-p u<\delta u / 2
$$

holds for all $u \geq M$. Then the term $I_{2}$ can be estimated as

$$
I_{2} \leq \frac{1}{M \pi} \int_{M}^{\infty} \exp (-\delta u / 2) d u \leq \frac{2 \exp (-M \delta / 2)}{M \delta \pi} .
$$

For $\beta \in(0,0.5]$, since $\cos (\beta \pi) \geq 0$, it follows that

$$
\begin{aligned}
I_{2} & \leq \frac{\exp \left(-M^{\beta} \cos (\beta \pi)\right)}{M \pi} \int_{M}^{\infty} \exp (-p u) d u \\
& \leq \frac{\exp \left(-M^{\beta} \cos (\beta \pi)\right)}{M \pi} \int_{M}^{\infty} \exp (-\delta u) d u \leq \frac{\exp \left(-M^{\beta} \cos (\beta \pi)-M \delta\right)}{M \delta \pi} .
\end{aligned}
$$

The following lemma and theorem analyse the corresponding situation for the complementary expression for $\phi(\beta, p)$ in (1.3).

Lemma 3.4. For a fixed $\beta \in(0,1)$, the integrand in the expression for $\phi(\beta, p)$ in (1.3),

$$
f_{2}(p, u)=\exp \left(-u^{\beta} \cos (\beta \pi / 2)\right) \cos \left(u^{\beta} \sin (\beta \pi / 2)-p u\right),
$$

is integrable on the domain $\left[p_{i}, p_{i+1}\right] \times(0, \infty)$ with $0<p_{i}<p_{i+1}<\infty$. Consequently, the order of integration in (3.3) can be changed, that is,

$$
a_{i}^{*}=\int_{p_{i}}^{p_{i+1}} \int_{0}^{\infty} f_{2}(p, u) d u d p=\int_{0}^{\infty} \int_{p_{i}}^{p_{i+1}} f_{1}(p, u) d p d u .
$$


Proof. Similarly to Lemma 3.2, it is sufficient to prove that the integral

$$
\int_{0}^{\infty}\left(\int_{p_{i}}^{p_{i+1}}\left|f_{2}(p, u)\right| d p\right) d u
$$

is finite. Since $\cos (\beta \pi / 2)>0$ for $\beta \in(0,1)$, on making change of variable $v=$ $u^{\beta} \cos (\beta \pi / 2)$, the following estimation proves the lemma:

$$
\begin{aligned}
\int_{0}^{\infty} \int_{p_{i}}^{p_{i+1}}\left|f_{2}(p, u)\right| d p d u & \leq \int_{0}^{\infty} \int_{p_{i}}^{p_{i+1}} \exp \left(-u^{\beta} \cos (\beta \pi / 2)\right) d p d u \\
& \leq\left(p_{i+1}-p_{i}\right) \int_{0}^{\infty} \exp \left(-u^{\beta} \cos (\beta \pi / 2)\right) d u \\
& =\frac{p_{i+1}-p_{i}}{\beta}(\cos (\beta \pi / 2))^{-1 / \beta} \Gamma(1 / \beta) .
\end{aligned}
$$

Using the expression for $\phi(\beta, p)$ in (1.3), the change in the order of integration in the formula for $a_{i}^{*}$ yields

$$
a_{i}^{*}=\bar{\Psi}\left(p_{i}\right)-\bar{\Psi}\left(p_{i+1}\right),
$$

where

$$
\bar{\Psi}(p)=\frac{1}{\pi} \int_{0}^{\infty} \frac{\exp \left(-u^{\beta} \cos (\beta \pi / 2)\right) \sin \left(u^{\beta} \sin (\beta \pi / 2)-p u\right)}{u} d u
$$

and the corresponding truncated integral becomes

$$
\bar{\Psi}_{\bar{\epsilon}, \bar{M}}(p)=\frac{1}{\pi} \int_{\bar{\epsilon}}^{\bar{M}} \frac{\exp \left(-u^{\beta} \cos (\beta \pi / 2)\right) \sin \left(u^{\beta} \sin (\beta \pi / 2)-p u\right)}{u} d u .
$$

Theorem 3.5. Take $\beta \in(0,1)$ fixed and $\bar{M}>\bar{\epsilon}>0$. For each given $\zeta>0$ and for all $p \leq \zeta$, the uniform truncation error between $\bar{\Psi}_{\epsilon, M}(p)$ and $\bar{\Psi}(p)$ satisfies

$$
\left|\bar{\Psi}_{\bar{\epsilon}, \bar{M}}(p)-\bar{\Psi}(p)\right| \leq \frac{1}{2} \bar{\epsilon}^{\beta}+\frac{1}{\pi} \zeta \bar{\epsilon}+\frac{1}{\beta \pi} \frac{\exp \left(-\bar{M}^{\beta} \cos (\beta \pi / 2)\right)}{\bar{M}^{\beta} \cos (\beta \pi / 2)} .
$$

Proof. Similarly to Theorem 3.3, the truncation integral can be divided into two parts:

$$
\begin{aligned}
\left|\bar{\Psi}_{\bar{\epsilon}, \bar{M}}(p)-\bar{\Psi}(p)\right| & \leq \frac{1}{\pi}\left(\int_{0}^{\bar{\epsilon}}+\int_{\bar{M}}^{\infty}\right)\left|\frac{\exp \left(-u^{\beta} \cos (\beta \pi / 2)\right) \sin \left(u^{\beta} \sin (\beta \pi / 2)-p u\right)}{u}\right| d u \\
& :=I_{1}+I_{2},
\end{aligned}
$$

where $I_{1}$ and $I_{2}$ represent the first and second integrals. Since $0<\cos (\beta \pi / 2)<1$ for $\beta \in(0,1)$, the maximum of $\exp \left(-u^{\beta} \cos (\beta \pi / 2)\right)$ on $(0, \bar{\epsilon})$ is attained at $u=0$. Thus, the first integral can be estimated as

$$
\begin{aligned}
I_{1} & \leq \frac{1}{\pi} \int_{0}^{\bar{\epsilon}}\left|\frac{\sin \left(u^{\beta} \sin (\beta \pi / 2)-p u\right)}{u}\right| d u \\
& \leq \frac{1}{\pi} \int_{0}^{\bar{\epsilon}}\left|\left(u^{\beta-1} \sin (\beta \pi / 2)-p\right)\right| d u \\
& \leq \frac{\sin (\beta \pi / 2)}{\beta \pi} \bar{\epsilon}^{\beta}+\frac{1}{\pi} \zeta \bar{\epsilon} \leq \frac{1}{2} \bar{\epsilon}^{\beta}+\frac{1}{\pi} \zeta \bar{\epsilon} .
\end{aligned}
$$


Considering the second integral, the change of variable $v=u^{\beta} \cos (\beta \pi / 2)$ yields the following estimate:

$$
\begin{aligned}
I_{2} & \leq \frac{1}{\pi} \int_{\bar{M}}^{\infty} \frac{\exp \left(-u^{\beta} \cos (\beta \pi / 2)\right)}{u} d u \leq \frac{1}{\beta \pi} \int_{\bar{M}^{\beta \cos (\beta \pi / 2)}}^{\infty} \frac{\exp (-v)}{v} d v \\
& \leq \frac{1}{\beta \pi \bar{M}^{\beta} \cos (\beta \pi / 2)} \int_{\bar{M}^{\beta \cos (\beta \pi / 2)}}^{\infty} \exp (-v) d v=\frac{\exp \left(-\bar{M}^{\beta} \cos (\beta \pi / 2)\right)}{\beta \pi \bar{M}^{\beta} \cos (\beta \pi / 2)} .
\end{aligned}
$$

Combining the estimates for $I_{1}$ and $I_{2}$ yields the required result.

Remark 3.6. Theorem 3.3 gives the uniform truncation error bound with $p \in[\delta, \infty)$ for an arbitrary given $\delta>0$, and the complementary Theorem 3.5 gives the uniform truncation error bound with $p \in[0, \zeta]$ for an arbitrary given $\zeta>0$. Together, they show that the truncation errors can be made uniformly small by using suitable $\epsilon, M, \bar{\epsilon}$ and $\bar{M}$. In the numerical results discussed below, the constants $\delta=\zeta=1$ are chosen, for which the formulae (1.2) and (1.3) are utilized for $p \leq \delta$ and $p>\delta$, respectively.

THEOREM 3.7. Let $\widetilde{a}_{i}^{*}$ denote the numerical approximation of $a_{i}^{*}$ when the formulae (1.2) and $(1.3)$ are used on $[\delta, \infty)$ and $[0, \delta]$, respectively, and let

$$
\widetilde{\sigma}(\beta, N)(t)=\sum_{i=1}^{N} \widetilde{a}_{i}^{*} \exp \left(-p_{i}^{*} t\right)
$$

be the corresponding numerical approximation of $\sigma(\beta, N)$ in $(3.4)$, where $p_{i}^{*} \in$ $\left(p_{i}, p_{i+1}\right)$ is independent of $t$. For each $t>0$, the error between the approximation $\widetilde{\sigma}(\beta, N)$ and the Kohlrausch function $\exp \left(-t^{\beta}\right)$ can be estimated as

$$
\left|\exp \left(-t^{\beta}\right)-\widetilde{\sigma}(\beta, N)\right| \leq \Upsilon_{0}(t)+\Upsilon_{\infty}(t)+\max _{j} a_{j}^{*} \exp \left(-p^{\#} t\right)+\frac{2 C(\delta) \exp \left(-p^{\#} t\right)}{1-\exp (-\rho t)}
$$

where $C(\delta)=\max \left\{C_{1}(\delta), C_{2}(\delta)\right\}$ is the larger uniform truncation bound in (3.7) or (3.9), with the choice $\delta=\zeta$, and $\rho>0$ is a constant such that $p_{i}^{*}>p_{i} \geq p^{\#}+(i-1) \rho$.

Proof. The proof is similar to that given by Loy and Anderssen [12]. The expression for the Kohlrausch function in (3.2) yields

$$
\begin{aligned}
& \left|\exp \left(-t^{\beta}\right)-\tilde{\sigma}(\beta, N)(t)\right| \\
& \quad=\left|\Upsilon_{0}(t)+\Upsilon_{\infty}(t)+\sum_{i=1}^{N} a_{i}^{*} \exp (-p(i, t))-\sum_{i=1}^{N} \tilde{a}_{i}^{*} \exp \left(-p_{i}^{*} t\right)\right| \\
& \quad=\left|\Upsilon_{0}(t)+\Upsilon_{\infty}(t)+\sum_{i=1}^{N} a_{i}^{*}\left(\exp (-p(i, t))-\exp \left(-p_{i}^{*} t\right)\right)+\sum_{i=1}^{N}\left(a_{i}^{*}-\tilde{a}_{i}^{*}\right) \exp \left(-p_{i}^{*} t\right)\right|
\end{aligned}
$$


For a uniform grid or a nonuniform grid with increasing intervals, there exists $\rho>0$ as specified in the statement of the theorem. Consequently,

$$
\begin{aligned}
& \left|\exp \left(-t^{\beta}\right)-\tilde{\sigma}(\beta, N)\right| \\
& \quad \leq \Gamma_{0}(t)+\Gamma_{\infty}(t)+\max _{j} a_{j}^{*} \exp \left(-p^{\#} t\right)+\max _{j}\left|a_{i}^{*}-\tilde{a}_{i}^{*}\right| \exp \left(-p^{\#} t\right) \sum_{i=0}^{N-1} \exp (-i \rho t) \\
& \quad \leq \Gamma_{0}(t)+\Gamma_{\infty}(t)+\max _{j} a_{j}^{*} \exp \left(-p^{\#} t\right)+\frac{2 C(\delta) \exp \left(-p^{\#} t\right)}{1-\exp (-\rho t)} .
\end{aligned}
$$

\section{Sums of exponentials approximations with midpoint estimates}

The uniform convergence result of Loy and Anderssen is valid for both uniform and nonuniform grids. Figure 1 shows that, for a fixed $\beta$, after attaining its unique maximum somewhere in $(0,1)$, the function $\phi(\beta, p)$ initially decays rapidly and then goes slowly to 0 monotonically. In addition, its derivative goes monotonically to 0 as $p$ tends to infinity. This implies that $\phi(\beta, p)$ is uniformly small for large $p$. Consequently, increasing grid intervals can be chosen so that the number of exponential terms is reduced, yet still giving a good approximation. Especially when $\beta$ is small, because of the long tail, a large $p^{\# \#}$ is needed in order to make $\Upsilon_{\infty}(t)$ small and obtain a good approximation. Many more terms would be required if a uniform grid was used.

For the approximations discussed below, the grid points were generated using the rule $p_{i+1}=c p_{i}$, with $c>1$ a scaling constant. The midpoint $p_{i}^{*}=\left(p_{i}+p_{i+1}\right) / 2$ was chosen as the estimator for $p(i, t)$ in (3.2).

4.1. The $\beta=0.5$ case When $\beta=0.5, \Psi(p)$ simplifies to become

$$
\Psi(p)=\frac{1}{\pi} \int_{0}^{\infty} \frac{\sin (\sqrt{u}) \exp (-p u)}{u} d u .
$$

This special case has been examined by Anderssen et al. [5]. The plots in Figure 2 compare the Kohlrausch function $\exp \left(-t^{1 / 2}\right)$ with sums of exponentials approximations.

4.2. The $\beta>0.5$ case The plots in Figure 3 compare the Kohlrausch function $\exp \left(-t^{0.8}\right)$ with sums of exponentials approximations. Since $\phi(\beta, p)$ becomes sharper and sharper as $\beta$ tends to 1 (shown in Figure 1), the corresponding $a_{i}^{*}$ is very small, except for grid intervals near 1 . In this situation, fewer exponential functions are required to obtain good approximations. Validation for this comment can be found in Section 5 (Table 2), where it is shown that, to achieve similar accuracies, the number of exponentials required decreases as $\beta$ increases.

4.3. The $0<\boldsymbol{\beta}<\mathbf{0 . 5}$ case In this situation, more and more exponentials are required to generate sufficiently accurate approximations to $\exp \left(-t^{\beta}\right)$ as $\beta$ decreases. This is a consequence of the initial rapid decay and the long tail for $\phi(\beta, p)$. The plots in Figures 4 and 5 compare the Kohlrausch functions $\exp \left(-t^{0.3}\right)$ and $\exp \left(-t^{0.1}\right)$, 


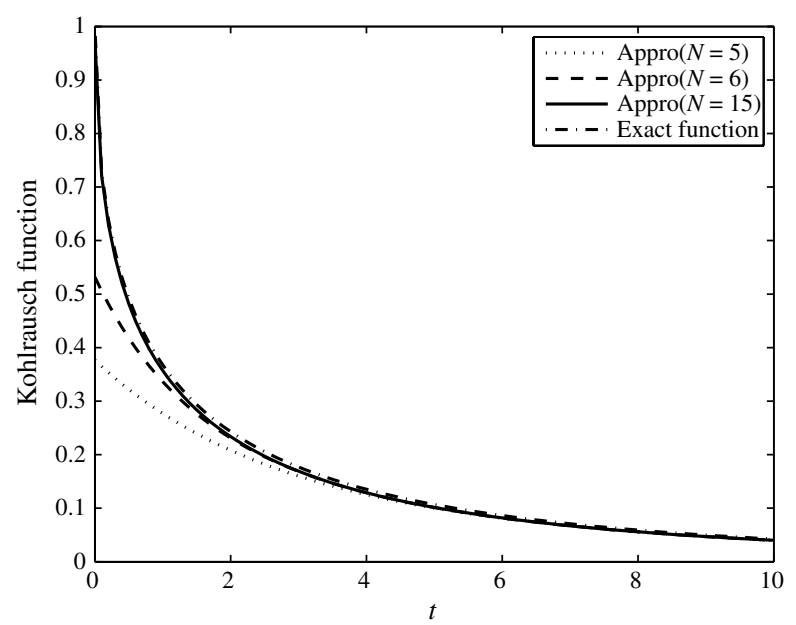

FIGURE 2. Comparison of the Kohlrausch function $\exp \left(-t^{0.5}\right)$ with sums of exponentials approximations with $p^{\#}=0.02, c=2$ and $N=5,6,15$. The corresponding $l^{2}$ and $l^{\infty}$ norms of the errors on the grid $t \in[0: 0.1: 10]$ are $\{0.915,0.623\},\{0.582,0.468\}$ and $\{0.069,0.022\}$.

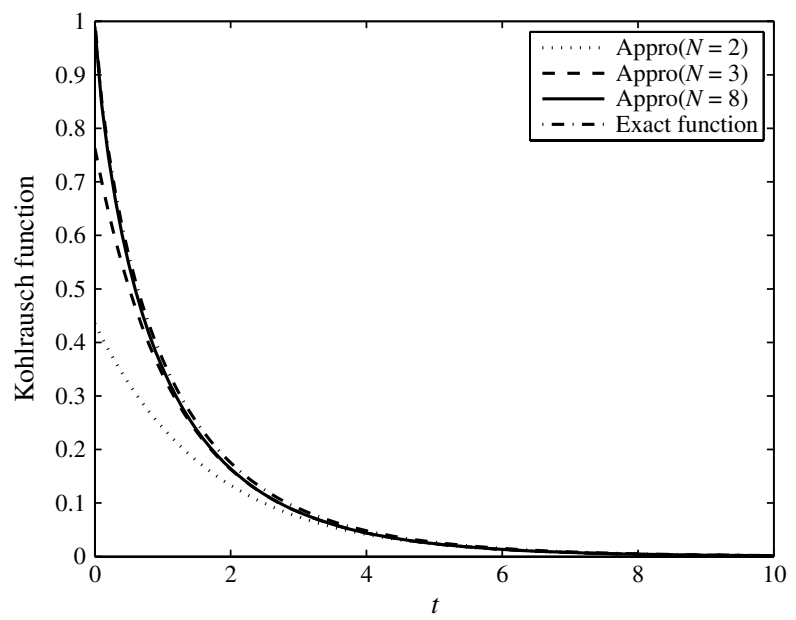

FIGURE 3. Comparison of the Kohlrausch function $\exp \left(-t^{0.8}\right)$ with sums of exponentials approximations with $p^{\#}=0.2, c=2$ and $N=2,3,8$. The corresponding $l^{2}$ and $l^{\infty}$ norms of the errors on the grid $t \in[0: 0.1: 10]$ are $\{1.046,0.564\},\{0.348,0.236\}$ and $\{0.074,0.017\}$.

respectively, with sums of exponentials approximations. For $\beta=0.1$, because of the long tail of $\phi(0.1, p)$, a larger scaling constant $c$ has been chosen to reduce the number of exponential terms.

REMARK 4.1. In the approximation for small $\beta$, the exponential terms $\exp \left(-p_{i}^{*} t\right)$ with $p_{i}^{*} \ll 1$ closely approximate the tail of the corresponding Kohlrausch function. The terms with $p_{i}^{*} \gg 1$ give essentially no improvement to the approximation of the tail, but give a major improvement to the approximation of the rapid decrease in the 


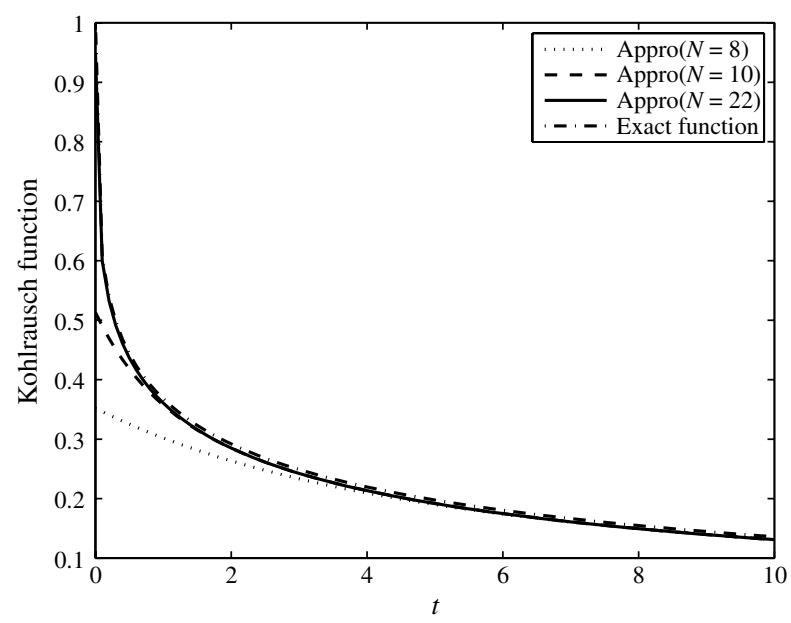

FIgure 4. Comparison of the Kohlrausch function $\exp \left(-t^{0.3}\right)$ with sums of exponentials approximations with $p^{\#}=0.002, c=2$ and $N=8,10,22$. The corresponding $l^{2}$ and $l^{\infty}$ norms of the errors on the grid $t \in[0: 0.1: 10]$ are $\{0.805,0.648\},\{0.514,0.487\}$ and $\{0.079,0.050\}$.

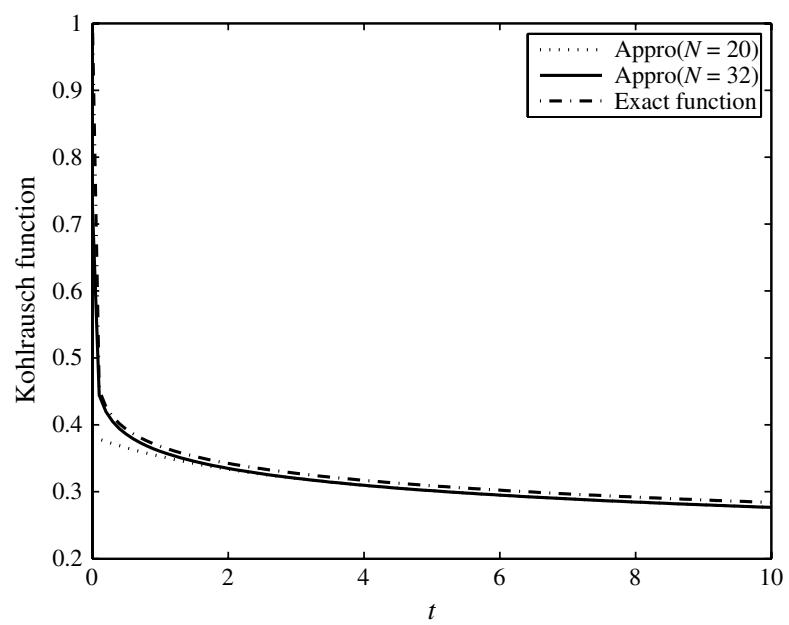

Figure 5. Comparison of the Kohlrausch function $\exp \left(-t^{0.1}\right)$ with sums of exponentials approximations with $p^{\#}=10^{-8}, c=2.5$ and $N=20,32$. The corresponding $l^{2}$ and $l^{\infty}$ norms of the errors on the grid $t \in[0: 0.1: 10]$ are $\{0.633,0.618\}$ and $\{0.284,0.274\}$.

neighbourhood of the origin $t=0$. For a larger scaling constant, $c=3.2$, and more exponential terms, $N=40$, a better approximation is obtained, with $l^{2}$ norm 0.115 and $l^{\infty}$ norm 0.064 for the error.

\section{A sharper estimate for the mean value points}

Figure 1 shows that, for a fixed $\beta \in(0,1)$, especially for a small $\beta \in(0,0.5)$, the function $\phi(\beta, p)$ grows to its maximum, then decreases, at first rapidly, and then with 
a long tail. This structure implies that, compared with the midpoint estimate, sharper estimates $p_{i}^{*}$ for the mean value points $p(i, t)$ on $\left(p_{i}, p_{i+1}\right)$ can be chosen to improve the approximation results.

Lemma 5.1. Suppose that the continuous nonnegative function $f$ is monotone decreasing on the interval $[a, b]$. Define $\xi \in(a, b)$ as the mean value point satisfying

$$
\exp (-\xi) \int_{a}^{b} f(p) d p=\int_{a}^{b} \exp (-p) f(p) d p
$$

and $\eta$ by

$$
\exp (-\eta)=\frac{1}{b-a} \int_{a}^{b} \exp (-p) d p=\frac{\exp (-a)-\exp (-b)}{b-a}
$$

Then $\xi \leq \eta<(a+b) / 2$.

Proof. Since

$$
\begin{aligned}
0 & =\int_{a}^{b} f(p)(\exp (-p)-\exp (-\xi)) d p \\
& =\int_{a}^{\xi} f(p)(\exp (-p)-\exp (-\xi)) d p+\int_{\xi}^{b} f(p)(\exp (-p)-\exp (-\xi)) d p \\
& \geq f(\xi) \int_{a}^{\xi}(\exp (-p)-\exp (-\xi)) d p+f(\xi) \int_{\xi}^{b}(\exp (-p)-\exp (-\xi)) d p \\
& =f(\xi)(\exp (-a)-\exp (-b)-(b-a) \exp (-\xi)),
\end{aligned}
$$

it follows that

$$
\exp (-\xi) \geq \frac{\exp (-a)-\exp (-b)}{b-a}=\exp (-\eta)
$$

Further, the strict convexity of $t \mapsto \exp (-t)$ gives

$$
\frac{\exp (-b)-\exp (-a)}{b-a}<-\exp \left(-\frac{a+b}{2}\right)
$$

The two inequalities (5.1) and (5.2) imply the result.

Applying Lemma 5.1 to the interval $\left[p_{i}, p_{i+1}\right]$ on which $\phi(\beta, p)$ is decreasing, recalling the formula (3.2) for the mean value point $p(i, t)$, and defining $\eta_{i}(t)$ by

$$
\left(p_{i+1}-p_{i}\right) \exp \left(-\eta_{i}(t) t\right)=\int_{p_{i}}^{p_{i+1}} \exp (-p t) d p,
$$

which can be rearranged to give

$$
\eta_{i}(t)=-\frac{1}{t} \ln \left(\frac{e^{-p_{i} t}-e^{-p_{i+1} t}}{\left(p_{i+1}-p_{i}\right) t}\right),
$$


it follows that, for $t>0$,

$$
p(i, t) \leq \eta_{i}(t)<\frac{p_{i}+p_{i+1}}{2}
$$

In our earlier construction of sums of exponentials approximations for $\exp \left(-t^{\beta}\right)$, we took $p_{i}^{*} \in\left(p_{i}, p_{i+1}\right)$, independent of $t$, to be the midpoint. The inequalities in (5.4) show that taking $p_{i}^{*}<\eta_{i}(t)$ on $\left(p_{i}, p_{i+1}\right)$ would be a better choice. The following algorithm gives a method for replacing the midpoint estimates with these sharper estimates.

- For a given $\beta$, find the first interval $\left[p_{s}, p_{s+1}\right]$ with $s \geq 1$ on which the function $\phi(\beta, p)$ is decreasing.

- For the time points $t_{j}=j h$, where $h$ denotes the grid step and $1 \leq j \leq n$, use (5.3) to calculate the entries $\eta_{k}\left(t_{j}\right), k=s, s+1, \ldots, N$, to generate the matrix $A_{\eta} \in \mathbb{R}^{n \times(N-s+1)}$.

- The sharper estimate $\xi_{i}$ for the mean value point $p(i, t)$ on the interval $\left[p_{i}, p_{i+1}\right]$ is then chosen as the minimum of the $i$ th column of the matrix $A_{\eta}$, that is,

$$
\xi_{i}=\min \left\{\eta_{i}\left(t_{1}\right), \ldots, \eta_{i}\left(t_{n}\right)\right\}, \quad s \leq i \leq N .
$$

For fixed $t>0$, it is not necessary to calculate all $\eta_{k}\left(t_{j}\right)$ with $k=s, s+1, \ldots, N$. In fact, choose two constants $C_{2}>C_{1}>0$. If there exist integers $1 \leq M_{1}(t) \leq M_{2}(t) \leq N$ such that

$$
C_{1} \leq p_{i} t \leq C_{2} \quad \text { or } \quad C_{1} \leq p_{i+1} t \leq C_{2}
$$

for $M_{1}(t) \leq i \leq M_{2}(t)$ then, for any $p_{i}^{* *} \in\left(p_{i}, p_{i+1}\right)$, independent of $t$, recalling that $a_{i}^{*}<1$ for all $i$, the following two estimates are derived:

$$
\begin{aligned}
\left|\sum_{i=s}^{M_{1}-1} a_{i}^{*}\left(\exp (-p(i, t) t)-\exp \left(-p_{i}^{* *} t\right)\right)\right| & \leq \sum_{i=s}^{M_{1}-1}\left(\exp \left(-p_{i} t\right)-\exp \left(-p_{i+1} t\right)\right) \\
& \leq \exp \left(-p_{s} t\right)-\exp \left(-p_{M_{1}-1} t\right) \\
& \leq 1-\exp \left(-C_{1}\right) \\
\left|\sum_{i=M_{2}+1}^{N} a_{i}^{*}\left(\exp (-p(i, t) t)-\exp \left(-p_{i}^{* *} t\right)\right)\right| & \leq \sum_{i=M_{2}+1}^{N}\left(\exp \left(-p_{i} t\right)-\exp \left(-p_{i+1} t\right)\right) \\
& \leq \exp \left(-p_{M_{2}+1} t\right)-\exp \left(-p^{\# \#} t\right) \\
& \leq \exp \left(-C_{2}\right)
\end{aligned}
$$

If $C_{1}$ is chosen suitably small and $C_{2}$ suitable large, the bounds in (5.5) and (5.6) will be small compared with the other error terms. This implies that, when considering sharper estimates for the mean value points for $t>0$, it is only necessary to consider those $M_{2}-M_{1}+1$ exponential terms between $M_{1}(t)$ and $M_{2}(t)$. For the others, any point on the interval $\left(p_{i}, p_{i+1}\right)$ can be used as the estimate of the mean value point $p(i, t)$. 


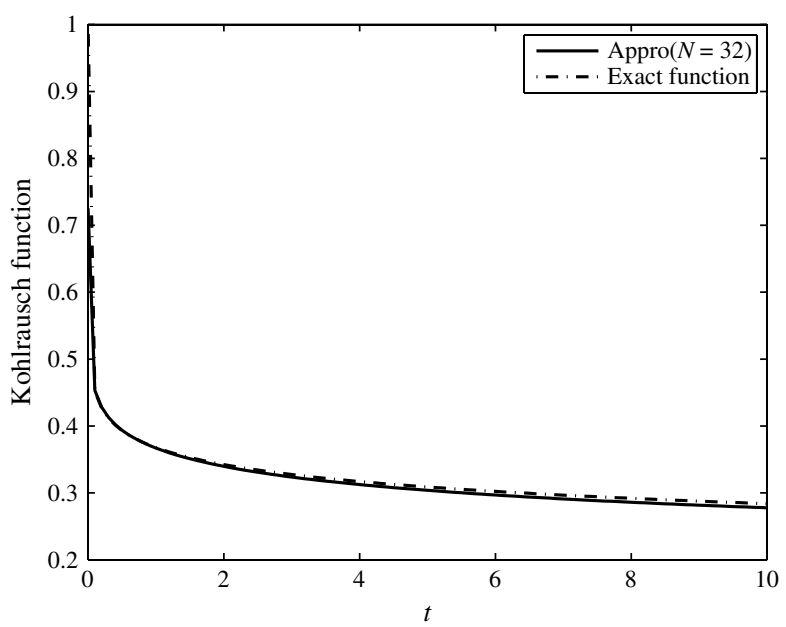

FIGURE 6. Comparison of the Kohlrausch function $\exp \left(-t^{0.1}\right)$ with sums of exponentials approximations for the modified form where $p^{\#}=10^{-8}, c=2.5$ and $N=32$. The corresponding $l^{2}$ and $l^{\infty}$ norms of the errors on the grid $t \in[0: 0.1: 10]$ are $\{0.260,0.274\}$.

REMARK 5.2. When $t$ is small, the sharper estimates for the mean value points only give a tiny improvement to the approximation, because both $M_{1}(t)$ and $M_{2}(t)$ go to $N$ as $t \rightarrow 0$, and if $t$ is very small, $M_{1}(t)$ and $M_{2}(t)$ may not exist. In this situation, the approximation error is controlled by the sum of the coefficients $\sum_{i=1}^{N} a_{i}^{*}$. The same thing happens when $t$ is very large: both $M_{1}(t)$ and $M_{2}(t)$ go to 1 or may not exist.

The algorithm can be simplified by taking the following lemma into account.

Lemma 5.3. Each $\eta_{i}(t)$ defined in (5.3) is a decreasing function of $t \in(0, \infty)$.

Proof. For convenience, let $\eta_{i}(t)=\eta(t), p_{i}=a$ and $p_{i+1}=b$. From (5.3), differentiation of $\eta(t)$ yields

$$
\dot{\eta}(t)=\frac{1}{t^{2}}\left(\ln \left(\frac{e^{-a t}-e^{-b t}}{(b-a) t}\right)+1-\frac{\left(b e^{-b t}-a e^{-a t}\right) t}{e^{-a t}-e^{-b t}}\right):=\frac{1}{t^{2}} g(t) .
$$

Differentiation of $g(t)$ then gives

$$
\dot{g}(t)=\frac{(b-a)^{2} e^{-(a+b) t} t^{2}-\left(e^{-a t}-e^{-b t}\right)^{2}}{\left(e^{-a t}-e^{-b t}\right)^{2} t}<0,
$$

because

$$
\exp (-(b+a) t / 2)<\exp (-\eta(t) t)=\frac{\exp (-a t)-\exp (-b t)}{t(b-a)} .
$$

Since $\exp (1+s)=1+s+o(s)$ for small $s$, it follows that

$$
g(t) \rightarrow 0 \quad \text { as } t \rightarrow 0
$$


TABLE 2. Representative sums of exponentials approximations for the Kohlrausch functions. The errors on the grid $t \in[0: 0.1: 10]$ for both midpoint estimates (MP) and sharper estimates (SE) are listed. The $a_{i}^{*}$ are approximated using (3.6) and (3.8), with $\epsilon=\bar{\epsilon}=10^{-12}$ and $M, \bar{M}$ suitably large to give consistent $l^{2}$ and $l^{\infty}$ errors.

\begin{tabular}{|c|c|c|c|c|c|}
\hline$\beta$ & $N$ & Nodes & $l^{2}$ & $l^{\infty}$ & Coefficients $a_{1}^{*}, \ldots, a_{N}^{*}$ \\
\hline 0.9 & 6 & $\begin{array}{l}p^{\#}=0.4 \\
p_{i+1}=2 p_{i}\end{array}$ & $\begin{array}{l}0.062(\mathrm{MP}) \\
0.043(\mathrm{SE})\end{array}$ & $\begin{array}{l}0.017(\mathrm{MP}) \\
0.010(\mathrm{SE})\end{array}$ & $\begin{array}{c}0.347,0.513,0.088,0.028,0.012 \\
0.005\end{array}$ \\
\hline 0.8 & 8 & $\begin{array}{l}p^{\#}=0.2 \\
p_{i+1}=2 p_{i}\end{array}$ & $\begin{array}{l}0.074 \\
0.046\end{array}$ & $\begin{array}{l}0.017 \\
0.013\end{array}$ & $\begin{array}{c}0.009,0.426,0.328,0.126,0.054 \\
0.026,0.014,0.007\end{array}$ \\
\hline 0.7 & 10 & $\begin{array}{l}p^{\#}=0.1 \\
p_{i+1}=2 p_{i}\end{array}$ & $\begin{array}{l}0.070 \\
0.041\end{array}$ & $\begin{array}{l}0.015 \\
0.013\end{array}$ & $\begin{array}{l}0.001,0.117,0.327,0.245,0.136 \\
0.074,0.041,0.024,0.014,0.008\end{array}$ \\
\hline 0.6 & 10 & $\begin{array}{l}p^{\#}=0.1 \\
p_{i+1}=2 p_{i}\end{array}$ & $\begin{array}{l}0.077 \\
0.051\end{array}$ & 0.028 & $\begin{array}{l}0.038,0.170,0.230,0.190,0.131 \\
0.085,0.055,0.035,0.023,0.015\end{array}$ \\
\hline 0.5 & 15 & $\begin{array}{l}p^{\#}=0.02 \\
p_{i+1}=2 p_{i}\end{array}$ & $\begin{array}{l}0.069 \\
0.049\end{array}$ & 0.022 & $\begin{array}{l}0.001,0.012,0.064,0.134,0.165 \\
0.155,0.127,0.096,0.070,0.051 \\
0.036,0.026,0.018,0.013,0.009\end{array}$ \\
\hline 0.4 & 17 & $\begin{array}{l}p^{\#}=0.01 \\
p_{i+1}=2 p_{i}\end{array}$ & $\begin{array}{l}0.074 \\
0.053\end{array}$ & 0.037 & $\begin{array}{c}0.003,0.017,0.047,0.083,0.110 \\
0.119,0.115,0.102,0.086,0.070 \\
0.056,0.044,0.034,0.026,0.020 \\
0.016,0.012\end{array}$ \\
\hline 0.3 & 22 & $\begin{array}{l}p^{\#}=0.002 \\
p_{i+1}=2 p_{i}\end{array}$ & $\begin{array}{l}0.079 \\
0.059\end{array}$ & 0.050 & $\begin{array}{c}0.003,0.009,0.021,0.037,0.054 \\
0.068,0.078,0.083,0.083,0.079 \\
0.072,0.065,0.057,0.049,0.041 \\
0.035,0.029,0.025,0.020,0.017 \\
0.014,0.011\end{array}$ \\
\hline 0.2 & 30 & $\begin{array}{l}p^{\#}=10^{-4} \\
p_{i+1}=2.3 p_{i}\end{array}$ & $\begin{array}{l}0.082 \\
0.053\end{array}$ & 0.037 & $\begin{array}{l}0.003,0.007,0.013,0.021,0.030 \\
0.040,0.049,0.056,0.060,0.063 \\
0.063,0.062,0.059,0.056,0.051 \\
0.047,0.042,0.037,0.033,0.029 \\
0.025,0.022,0.019,0.016,0.014 \\
0.012,0.010,0.009,0.008,0.006\end{array}$ \\
\hline 0.1 & 40 & $\begin{array}{l}p^{\#}=10^{-8} \\
p_{i+1}=3.2 p_{i}\end{array}$ & $\begin{array}{l}0.115 \\
0.085\end{array}$ & 0.064 & $\begin{array}{l}0.001,0.003,0.005,0.008,0.012 \\
0.016,0.020,0.024,0.029,0.032 \\
0.036,0.038,0.040,0.042,0.043 \\
0.043,0.043,0.042,0.040,0.039 \\
0.037,0.035,0.039,0.031,0.029 \\
0.027,0.024,0.022,0.020,0.018 \\
0.017,0.015,0.014,0.012,0.011 \\
0.010,0.009,0.008,0.006,0.003\end{array}$ \\
\hline
\end{tabular}

Together, the estimates (5.7) and (5.8) imply that $g(t)<0$, or equivalently, $\dot{\eta}(t)<0$, which proves the lemma. 
Using Lemma 5.3, the above algorithm simplifies.

- For a given $\beta$, find the first interval $\left[p_{s}, p_{s+1}\right]$ with $s \geq 1$ on which the function $\phi(\beta, p)$ is decreasing.

- For $t_{n}>0$, which is the last time point, check whether there exists an interval $\left[p_{l}, p_{l+1}\right]$ with $s \leq l \leq N$ such that

$$
C_{1} \leq p_{l} t_{n} \leq C_{2} \quad \text { or } \quad C_{1} \leq p_{l+1} t_{n} \leq C_{2} .
$$

If it exists, calculate $\eta_{l}\left(t_{n}\right)$, and choose $\xi_{l}=\eta_{l}\left(t_{n}\right)$ as the sharper estimate for the mean value point $p(l, t)$ to replace the midpoint estimate. Lemma 5.3 ensures that $\eta_{l}\left(t_{n}\right)$ is the minimum value of $\eta_{l}$ for all $t_{j}$. Otherwise, keep the midpoint estimate.

- Repeat the procedure for $t_{n-1}, \ldots, t_{1}$ to decide whether sharper estimates can be taken on the remaining intervals.

Using these sharper estimates for the mean value point, smaller $l^{2}$ norms on the grid $t \in[0: 0.1: 10]$ are obtained. However, for small $\beta$, such adjustments do not improve the error for the $l^{\infty}$ norm which is located near $t=0$. The plots in Figure 6 approximate the Kohlrausch function $\exp \left(-t^{0.1}\right)$ using the sharper estimates. The results compare favourably with those in Figure 5 with midpoint estimates for the mean value points.

The detailed results for these sharper estimates for the mean value points for different values of $\beta \in[0.1: 0.1: 0.9]$ are given in Table 2 , with scaling constants, choices of $p^{\#}$, numbers of exponential terms $N$ and coefficients $\left\{a_{i}^{*}\right\}_{i=1}^{N}$. The associated $l^{2}$ and $l^{\infty}$ errors on the grid $t \in[0: 0.1: 10]$ for both midpoint estimates and sharper estimates are tabulated. The sharper estimates give better approximations for the Kohlrausch functions.

\section{Acknowledgements}

The research of M. Zhong is supported by the National Natural Science Foundation of China (No. 11101093) and a Chinese Scholarship Council scholarship. M. Zhong also gratefully acknowledges the support, advice and mentoring of Professor Jin Cheng (Fudan University, China).

\section{References}

[1] R. S. Anderssen, A. R. Davies and F. R. de Hoog, "On the interconversion integral equation for relaxation and creep", ANZIAM J. 48 (2007) C34-C266; http://journal.austms.org.au/ojs/index.php/ANZIAMJ/article/view/64.

[2] R. S. Anderssen, A. R. Davies and F. R. de Hoog, "On the sensitivity of interconversion between relaxation and creep”, Rheol. Acta 47 (2008) 159-167; doi:10.1007/s00397-007-0223-6.

[3] R. S. Anderssen, A. R. Davies and F. R. de Hoog, "On the Volterra integral equation relating creep and relaxation", Inverse Problems 24 (2008) 035009; doi:10.1088/0266-5611/24/3/035009.

[4] R. S. Anderssen, M. P. Edwards, S. A. Husain and R. J. Loy, "Sums of exponentials approximations for the Kohlrausch function", in: MODSIM2011, 19th International Congress on Modelling and Simulation (eds F. Chan, D. Marinova and R. S. Anderssen) (Modelling and Simulation Society of Australia and New Zealand, 2011), 263-369; http://www.mssanz.org.au/modsim2011/A3/anderssen.pdf. 
[5] R. S. Anderssen, S. A. Husain and R. J. Loy, "The Kohlrausch function: properties and applications", ANZIAM J. 45 (2004) C800-C816;

http://journal.austms.org.au/ojs/index.php/ANZIAMJ/article/view/924.

[6] M. N. Berberan-Santos, "Analytical inversion of the Laplace transform without contour integration: application to luminescence decay laws and other relaxation functions", J. Math. Chem. 38 (2005) 165-173; doi:10.1007/s10910-005-4961-3.

[7] M. N. Berberan-Santos, E. N. Bodunov and B. Valeur, "Mathematical functions for the analysis of luminescence decays with underlying distributions 1 . Kohlrausch decay function (stretched exponential)", Chem. Phys. 315 (2005) 171-182; doi:10.1016/j.chemphys.2005.04.006.

[8] P.-G. de Gennes, "Relaxation anomalies in linear polymer melts", Macromolecules 35 (2002) 3785-3786; doi:10.1021/ma012167y.

[9] W. Feller, An introduction to probability theory and its applications, Volume II (Wiley, New York, 1966).

[10] C. P. Lindsey and G. D. Patterson, "Detailed comparison of the Williams-Watts and ColeDavidson functions", J. Chem. Phys. 73 (1980) 3348-3357; doi:10.1063/1.440530.

[11] Y. Liu, "Approximation by Dirichlet series with nonnegative coefficients", J. Approx. Theory 112 (2001) 226-234; doi:10.1006/jath.2001.3589.

[12] R. J. Loy and R. S. Anderssen, On the construction of Dirichlet series approximations for completely monotone functions, Math. Comp. to appear; doi:10.1090/S0025-5718-2013-02725-1.

[13] J. R. Macdonald, "Accurate fitting of immittance spectroscopy frequency-response data using the stretched exponential model", J. Non-Cryst. Solids 212 (1997) 95-116; doi:10.1016/S0022-3093(96)00657-6.

[14] J. R. Macdonald, "Surprising conductive- and dielectric-system dispersion differences and similarities for two Kohlrausch-related relaxation-time distributions", J. Phys.: Condens. Matter. 18 (2006) 629-644; doi:10.1088/0953-8984/18/2/019.

[15] E. W. Montroll and J. T. Bendler, "On Lévy (or stable) distributions and the Williams-Watts model of dielectric relaxation", J. Stat. Phys. 34 (1984) 129-162; doi:10.1007/BF01770352.

[16] K. L. Ngai and C. M. Roland, "Development of cooperativity in the local segmental dynamics of (poly)vinylacetate: synergy of thermodynamics and intermolecular coupling", Polymer 43 (2002) 567-573; doi:10.1016/S1089-3156(01)00011-3.

[17] A. Nikonov, A. R. Davies and I. Emri, "The determination of creep and relaxation functions from a single experiment", J. Rheol. 49 (2005) 1193-1211; doi:10.1122/1.2072027.

[18] K. A. Penson and K. Górska, "Exact and explicit probability densities for one-sided Lévy stable distributions", Phys. Rev. Lett. 105 (2010) 210604; doi:10.1103/PhysRevLett.105.210604.

[19] H. Pollard, "The representation of $e^{-x^{\lambda}}$ as a Laplace integral", Bull. Amer. Math. Soc. 52 (1946) 908-910; doi:10.1090/S0002-9904-1946-08672-3.

[20] R. Rambousky, M. Weiss, H. Mysz, M. Moske and K. Samwer, "Structural relaxation and viscous flow in amorphous $\mathrm{ZrAlCu}$ above and below the glass transition temperature", Mater. Sci. Forum 225-227 (1996) 83-88; doi:10.4028/www.scientific.net/MSF.225-227.83.

[21] N. Sasaki, Y. Nakayama, M. Yoshikawa and A. Enyo, "Stress relaxation function of bone and bone collagen", J. Biomechanics 26 (1993) 1369-1376; doi:10.1016/0021-9290(93)90088-V.

[22] M. F. Shlesinger and E. W. Montroll, "On the Williams-Watts function of dielectric relaxation", Proc. Natl. Acad. Sci. 81 (1984) 1280-1283; doi:10.1073/pnas.81.4.1280. 